\title{
Electro-acupuncture ameliorates cognitive impairment via improvement of brain-derived neurotropic factor-mediated hippocampal synaptic plasticity in cerebral ischemia-reperfusion injured rats
}

\author{
RUHUI LIN ${ }^{1 *}$, XIAOJIE LI $^{2 *}$, WEILIN LIU ${ }^{3}$, WENLIE CHEN ${ }^{1}$, KUNQIANG YU $^{4}$, CONGKUAI ZHAO $^{4}$, \\ JIA HUANG ${ }^{3}$, SHANLI YANG ${ }^{4}$, HONGWEI PENG $^{4}$, JING TAO $^{3}$ and LIDIAN CHEN ${ }^{3}$ \\ ${ }^{1}$ Academy of Integrative Medicine Biomedical Research Center; ${ }^{2}$ Fujian Rehabilitation Tech Co-innovation Center; \\ ${ }^{3}$ College of Rehabilitation Medicine; ${ }^{4}$ Fujian Key Laboratory of Rehabilitation Technology, \\ Fujian University of Traditional Chinese Medicine, Fuzhou, Fujian 350122, P.R. China
}

Received February 19, 2016; Accepted February 1, 2017

DOI: $10.3892 /$ etm.2017.4750

\begin{abstract}
A previous study by our group found that electro-acupuncture (EA) at the Shenting (DU24) and Baihui (DU20) acupoints ameliorates cognitive impairment in rats with cerebral ischemia-reperfusion (I/R) injury. However, the precise mechanism of action has remained largely unknown. The present study investigated whether brain-derived neurotropic factor (BDNF) mediates hippocampal synaptic plasticity as the underlying mechanism. Rats were randomly divided into three groups: The sham operation control (Sham) group, the focal cerebral ischemia-reperfusion (I/R) group, and the I/R with EA treatment (I/R+EA) group. The I/R+EA group received EA treatment at the Shenting (DU24) and Baihui (DU20) acupoints after the operation. EA treatment was found to ameliorate neurological deficits $(\mathrm{P}<0.05)$ and reduce the cerebral infarct volume $(\mathrm{P}<0.01)$. In addition, EA improved
\end{abstract}

Correspondence to: Mrs. Jing Tao or Professor Lidian Chen, College of Rehabilitation Medicine, Fujian University of Traditional Chinese Medicine, 1 Qiuyang Road, Fuzhou, Fujian 350122, P.R. China

E-mail: 369049101@qq.com

E-mail: cld@fjtcm.edu.cn

*Contributed equally

Abbreviations: EA, electro-acupuncture; MCAO, middle cerebral artery occlusion; I/R, ischemia-reperfusion; BDNF, brain-derived neurotrophic factor; TrkB, tropomyosin receptor kinase B; PSD-95, postsynaptic density protein-95; ICA, internal carotid artery; CBF, cerebral blood flow; MRI, magnetic resonance imaging; ANOVA, analysis of variance

Key words: electro-acupuncture, cognitive impairment, synaptic plasticity, cerebral ischemia-reperfusion, brain-derived neurotropic factor cognitive function in cerebral I/R-injured rats $(\mathrm{P}<0.05)$. Furthermore, EA treatment promoted synaptic plasticity. Simultaneously, EA increased the hippocampal expression of BDNF, its high-affinity tropomyosin receptor kinase B (TrkB) and post-synaptic density protein-95 (PSD-95) in the rats with cerebral I/R injury. Collectively, the findings suggested that BDNF-mediated hippocampal synaptic plasticity may be one mechanism via which EA treatment at the Shenting (DU24) and Baihui (DU20) acupoints improves cognitive function in cerebral I/R injured rats.

\section{Introduction}

In 2011, an estimated 795,000 individuals experienced a stroke event in the United States of America (1). Stroke is one of the most common causes of cognitive impairment (2-4). According to different subtypes and diagnostic criteria of stroke, $17-92 \%$ of patients present with cognitive impairment 3 months after a stroke (5). Cognitive impairment can cause a variety of symptoms, such as a decline in attention, memory and problem-solving capability (6); therefore, prevention and treatment of stroke with cognitive impairment has become a priority in the global health sector.

Acupuncture at the Shenting (DU24) and Baihui (DU20) acupoints has been used in China to treat cognitive impairment for thousands of years. They are located on a branch of the Du Meridian that runs over the head, which are associated with cognitive function in Traditional Chinese Medicine. Previous studies by our group have confirmed the efficacy of electro-acupuncture (EA) on the Shenting (DU24) and Baihui (DU20) acupoints in the rehabilitation of cognitive impairment in experimental settings $(7,8)$.

Although the pathogenic mechanisms of post-stoke cognitive impairment are complex, decreased hippocampal synaptic plasticity has been suggested to be one of the key elements $(9,10)$. Modulation of synaptic plasticity is highly controlled by brain-derived neurotropic factor (BDNF) protein binding to specific tropomyosin receptor kinase B (TrkB) 
receptors $(11,12)$. BDNF is a member of the neurotrophin family $(13,14)$, which is known to not only promote the activity of nerve growth, but also to modulate synaptic plasticity (15). For instance, numerous studies have indicated that BDNF enhances hippocampal synaptic plasticity by inducing local protein synthesis in the pre-synaptic terminal and post-synaptic dendrites of rats with cerebral ischemic injury $(16,17)$. EA has been proved to protect the synaptic ultrastructure and promote the expression of $\mathrm{P} 38$, growth-associated protein- 43 and BDNF in the ischemic cerebral cortex (18). However, the effects of EA on BDNF-mediated hippocampal synaptic plasticity and spatial memory after brain ischemia have remained elusive.

The present study thus investigated the effects of EA at the Shenting (DU24) and Baihui (DU20) acupoints on spatial memory performance and BDNF-mediated hippocampal synaptic plasticity by demonstrating the expression of hippocampal BDNF, TrkB and post-synaptic density protein-95 (PSD-95), as well as the synaptic morphology and structure in cerebral I/R-injured rats.

\section{Materials and methods}

Animals and grouping. A total of 30 healthy adult male Sprague-Dawley rats (weight, 260-300 g; 3 months old), were provided by Fujian University of Traditional Chinese Medicine Laboratory Animal Center [Fuzhou, China; Laboratory Animal Use Certificate no. SYXK (FJ) 2005-004] and housed under pathogen-free conditions at a temperature of $22 \pm 1^{\circ} \mathrm{C}$, with $50 \%$ humidity, under a 12 -h light/dark cycle with free access to food and water. All experiments were approved by the Institutional Animal Care and Use Committee of Fujian University of Traditional Chinese Medicine (Fuzhou, China). Rats were randomly divided into three groups $(\mathrm{n}=10)$ as follows: i) Sham operation control group (Sham group), ii) cerebral ischemia-reperfusion $(I / R)$ control group $(I / R$ group) and iii) $\mathrm{I} / \mathrm{R}$ with EA treatment group (I/R+EA group).

Middle cerebral artery occlusion (MCAO) model of focal cerebral ischemia. MCAO was used to establish a cerebral I/R-injured rat model according to the method by Longa et al (19). In brief, rats were anesthetized with $10 \%$ chloral hydrate $(300 \mathrm{mg} / \mathrm{kg}$; Beichen Founder Reagent Plant, Tianjin, China) by intraperitoneal injection. The left common carotid artery, left internal carotid artery (ICA) and the left external carotid artery were then carefully exposed following a midline neck incision. Subsequently, 18-22 $\mathrm{mm}$ of nylon surgical thread was inserted into the ICA in order to block the MCA until the blunted distal end met resistance. After $1.5 \mathrm{~h}$ of occlusion, the thread was removed to allow reperfusion of the MCA area. In I/R animals, a Laser Doppler Flowmetry system (BIOPAC Systems, Goleta, CA, USA) attached to the surface of the skull over the ipsilateral cortex was used to measure regional cerebral blood flow (CBF) to confirm consistent and similar levels of ischemic induction. During the surgical procedure, a reduction in $\mathrm{CBF}$ by $\geq 80 \%$ was considered to indicate successful modeling. Throughout the surgical procedure, the rectal temperature of the rats was maintained at $\sim 37^{\circ} \mathrm{C}$.

EA treatment. In the I/R+EA group, rats were administered EA for $30 \mathrm{~min}$ once daily for 7 days. The treatment was performed $24 \mathrm{~h}$ following the surgery and was continued for 7 days after the operation. The acupuncture needles $(0.3 \mathrm{~mm}$ diameter) were inserted at a depth of 2-3 mm into the heads of the rats at the Shenting (DU24) and Baihui (DU20) acupoints. Stimulation was then performed using an EA apparatus (Model SDZ-V; Suzhou Hua Tuo Medical Instrument Co., Ltd., Suzhou, China) with the stimulation parameters set as follows: $2 / 10 \mathrm{~Hz}$ at $1-3 \mathrm{~mA}$, dispersed for $30 \mathrm{~min}$ once daily. The Sham and I/R groups did not undergo any EA treatment.

Neurological deficit scoring. The neurological deficit score was determined at $1.5 \mathrm{~h}$ after I/R and 7 days after EA treatment to confirm successful MCAO (20) using the following scoring system: 0 , no neurological deficit; 1 , failure to fully extend the right forepaw (mild deficits); 2 , circling to the right (moderate deficits); 3 , falling to the right (moderate deficits); 4 , loss of walking (severe deficits). Rats with neurological deficit scores of 1-3 were included in the experiments.

Magnetic resonance imaging (MRI). A total of 7 days after EA treatment, rats were anesthetized with $1.5 \%$ isoflurane (Shenzhen Reward Life Technologies, Co., Ltd., Shenzheng, China and placed prone in a rat cradle, with the head restrained using ear and tooth bars to limit movement, and a linear surface receiver coil was then placed above the head of the rats. T2-weighted imaging by 7.0 Tesla MRI (BioSpec 70/20; Bruker, Billerica, MA, USA) was used to evaluate the full extent of the infarct using a Rapid Acquisition with Relaxation Enhancement T2 sequence (echo time, $33 \mathrm{msec}$; recovery time, $2,738 \mathrm{msec}$; scan time, $5.85 \mathrm{~min}$; field of view, 30x30 mm; 24 contiguous slices of $0.8 \mathrm{~mm}$ thickness). The infarct volume was quantified with Image J software 1.42q (National Institutes of Health, Bethesda, MD, USA) as a percentage of the total brain volume.

Morris water maze test. All of the rats were subjected to the Morris water maze test from the 3rd day after EA treatment to assess spatial learning ability and memory $(8,21)$. The water maze apparatus (Chinese Academy of Sciences, Beijing, China) included a circular pool (diameter, $120 \mathrm{~cm}$; depth, $50 \mathrm{~cm}$ ) filled with water (temperature, $25 \pm 2^{\circ} \mathrm{C}$; depth, $30 \mathrm{~cm}$ ). The pool was divided into four equal quadrants and a video camera attached to a computer was placed above the center of the pool to record each trial. A submerged safe platform was located in the pool ( $2 \mathrm{~cm}$ below water surface; $6 \mathrm{~cm}$ diameter in a fixed position).

Orientation navigation and space exploration trials were mainly included in the Morris water maze test. The orientation navigation trial consisted of 4 swims daily for 4 days (days 3-6). During this trial, each rat was allowed a $90-\mathrm{sec}$ swim to find the platform and was permitted to remain on it for at least $3 \mathrm{sec}$ with the swimming duration measured. If the rat could not find the platform within $90 \mathrm{sec}$, it was gently placed on it for a 10 -sec rest. The result of the swimming duration in the four quadrants was evaluated for each rat per day. The space exploration trial was performed on day 7 with the platform removed and each rat was allowed a free 90 -sec swim, during which the number of times each of the rats found the former location of the platform was recorded. After the trials, rats were dried with a hair drier and returned to their cages. 
Table I. Effect of EA on neurological deficit scores.

\begin{tabular}{lcc}
\hline Group & $1.5 \mathrm{~h}$ after I/R & 7 days after EA \\
\hline Sham & 0 & 0 \\
I/R & $2.10 \pm 0.74$ & $1.90 \pm 0.74$ \\
I/R+EA & $2.00 \pm 0.82$ & $1.20 \pm 0.63^{\mathrm{a}}$
\end{tabular}

Values are expressed as the mean \pm standard deviation $(\mathrm{n}=10) .{ }^{\mathrm{a}} \mathrm{P}<0.05$ vs. the I/R group. I/R, ischemia-reperfusion; EA, electro-acupuncture.

Electron microscopy. Hippocampal tissues for electron microscopy were pre-fixed for $24 \mathrm{~h}$ at $4^{\circ} \mathrm{C}$ in $3 \%$ glutaraldehyde, post-fixed for $2 \mathrm{~h}$ in $1 \%$ osmium tetroxide, dehydrated and flat-embedded in Araldite resin. The resin was baked as follows: $35^{\circ} \mathrm{C}$ for $24 \mathrm{~h}, 45^{\circ} \mathrm{C}$ for $24 \mathrm{~h}$ and $60^{\circ} \mathrm{C}$ for $48 \mathrm{~h}$. Subsequently, they were sliced into ultrathin sections of $90 \mathrm{~nm}$. After uranyl acetate and lead citrate double staining, the sections were observed under an electron microscope at a magnification of x50,000 (H-7650; Hitachi, Tokyo, Japan).

Western blot analysis. The left cerebral hippocampal tissues were collected and triturated in a radioimmunoprecipitation assay buffer (Fansbio, Guangdong, China), and the proteins were quantified using a bicin-choninic acid assay (Pierce; Thermo Fisher Scientific, Inc., Waltham, MA, USA) Subsequently, protein samples $(50 \mu \mathrm{g})$ were separated by $10 \%$ sodium dodecyl sulfate polyacrylamide gel electrophoresis. Proteins were then transferred onto polyvinylidene difluoride membranes, which were blocked for $2 \mathrm{~h}$ with $5 \%$ non-fat dried milk at room temperature. Blots were probed with antibodies targeted against BDNF (ab108319; 1:2,000 dilution; Abcam, Cambridge, MA, USA), TrkB (4603; 1:1,000 dilution; Cell Signaling Technology, Inc., Beverly, MA, USA), PSD-95 (3409; 1:1,000 dilution; Cell Signaling Technology, Inc.) and $\beta$-actin (HC201-01; 1:5,000 dilution; TransGen Biotech, Beijing, China) at $4{ }^{\circ} \mathrm{C}$ overnight, followed by incubation with horseradish peroxidase-conjugated secondary antibody $(1: 5,000$ dilution; TransGen Biotech, Beijing, China) for $1 \mathrm{~h}$ at $20^{\circ} \mathrm{C}$. All blots were visualized by enhanced chemiluminescence (BeyoECLPlus; Beyotime, Shanghai, China) and analyzed with the Bio-Image Analysis System (Bio-Rad Laboratories, Hercules, CA, USA).

Statistical analysis. All data were analyzed using the SPSS 18.0 software package (SPSS, Inc., Chicago, IL, USA). Values are expressed as the mean \pm standard deviation. Student's t-test, the Mann-Whitney U test, the Kruskal-Wallis H test, one-way analysis of variance (ANOVA) and repeated measures ANOVA were used. $\mathrm{P}<0.05$ was considered to indicate a statistically significant difference.

\section{Results}

EA treatment reduces neurological deficits and infarct volume in cerebral I/R-injured rats. Table I shows that at $1.5 \mathrm{~h}$ after cerebral I/R injury, neurological deficit scores of rats in the $\mathrm{I} / \mathrm{R}$ group were not significantly different to those of rats in the I/R+EA group ( $\mathrm{P}>0.05)$. However, on the 7 th day after EA treatment, the neurological deficit score in the $\mathrm{I} / \mathrm{R}+\mathrm{EA}$ group was significantly decreased compared with that in the I/R group $(\mathrm{P}<0.05)$. To further verify these results, the effect of EA on cerebral infarction was assessed by MRI (Fig. 1A). As shown in Fig. 1B, EA treatment reduced the cerebral infarct volume. The total infarct volumes in the $\mathrm{I} / \mathrm{R}$ and $\mathrm{I} / \mathrm{R}+\mathrm{EA}$ groups were $28.02 \pm 2.91$ and $21.86 \pm 3.11 \%$ of the total brain volume $(\mathrm{P}<0.01)$.

EA treatment attenuates cognitive impairment in cerebral $I / R$-injured rats. In the orientation navigation test, the I/R group showed a longer escape latency (the time it took the rats to find the platform) compared with the Sham group. Furthermore, the escape latency in the I/R+EA group was significantly reduced compared with that in the I/R group $(\mathrm{P}<0.05$; Fig. $2 \mathrm{~A}$ and $\mathrm{B})$. Moreover, in the space exploration test, the number of times rats in the I/R group passed through the original position of the platform was lower than that in the Sham group. In the I/R+EA group, the number of times the rats crossed the position of the platform was significantly increased compared with that in the I/R group ( $\mathrm{P}<0.01$; Fig. $2 \mathrm{C})$.

EA treatment improves the synaptic ultrastructure in cerebral $I / R$-injured rats. In the Sham group, axons were observed in the hippocampal neurons, which had abundant mitochondrial crista. Moreover, a large number of dendrites also existed in the hippocampal neurons, with abundant synapses. Furthermore, there were abundant synaptic vesicles in the preand post-synaptic membrane and synaptic clefts were clear (Fig. 3A). In the I/R group, only small amounts of dendrites and axons existed. The vacuolization of mitochondria with destruction of crista and the structure of synapses was disordered and damaged obviously (Fig. 3B). In the I/R+EA group, the vacuolization of mitochondria in the axons was not obvious compared with the I/R group. Moreover, synaptic clefts were identifiable and certain synaptic structures were similar to those in the Sham group (Fig. 3C).

Effects of EA treatment enhance the expression of BDNF and its receptor TrkB in cerebral I/R-injured rats. In the I/R group, the expression levels of BDNF and its receptor TrkB in the left hippocampus were significantly higher compared with the Sham group $(\mathrm{P}<0.01)$. Furthermore, the expression levels of BDNF and its receptor TrkB were significantly increased in the I/R+EA group compared with the I/R group $(\mathrm{P}<0.01$; Fig. 4).

EA treatment attenuates $I / R$-induced decreases of left hippocampal PSD-95 expression in rats. In the I/R group, the expression of PSD-95 in the left hippocampus was significantly lower compared to that in the Sham group $(\mathrm{P}<0.01)$. By contrast, the I/R+EA group had a higher relative content of PSD-95 protein than the I/R group $(\mathrm{P}<0.01$; Fig. 5$)$.

\section{Discussion}

The main findings of the present study were that EA treatment at the Shenting (DU24) and Baihui (DU20) acupoints improved the learning ability and memory of rats after brain ischemia. 
A
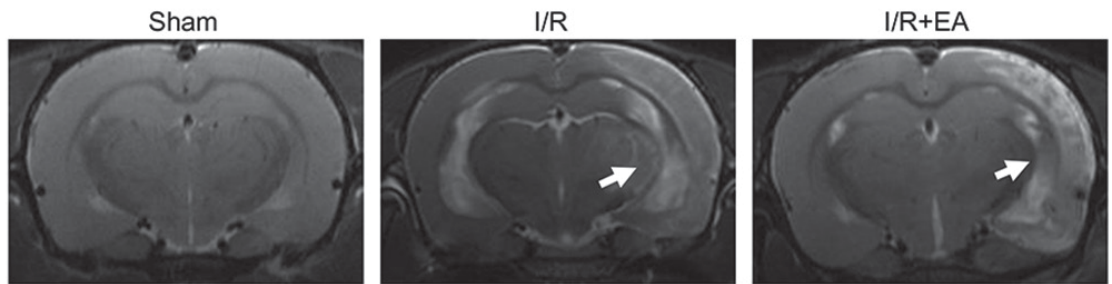

B

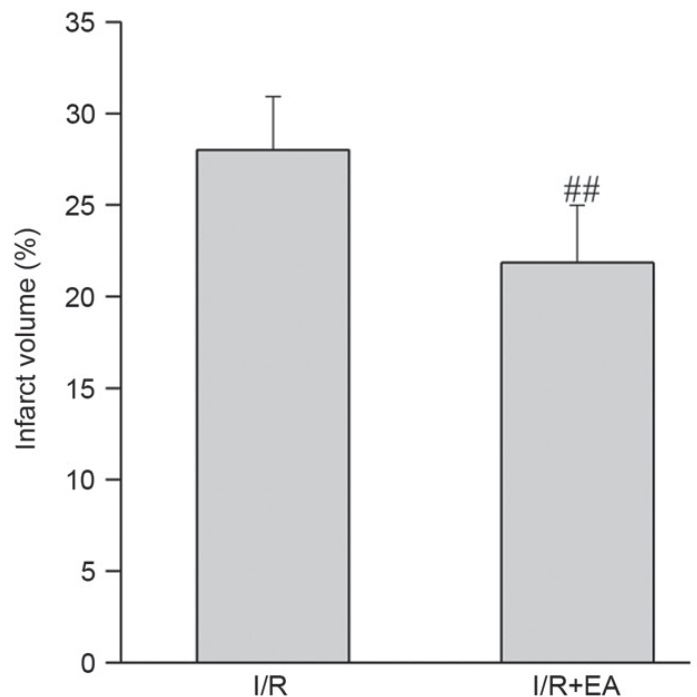

Figure 1. Effect of EA on infarct volume. (A) T2-weighted nuclear magnetic imaging indicated cerebral infarct volume of I/R and I/R+EA groups. Red arrows indicate the infarct site. (B) Bar graph showing the infarct volume, which was quantified as a percentage of the total brain volume in each group ( $\mathrm{n}=10$ ). ${ }^{\# \#} \mathrm{P}<0.01$ vs. the $\mathrm{I} / \mathrm{R}$ group. I/R, ischemia-reperfusion; EA, electro-acupuncture.

A

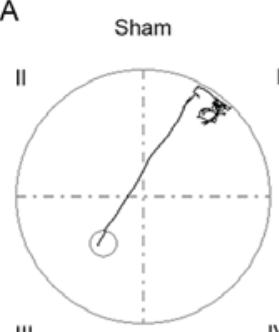

IV III
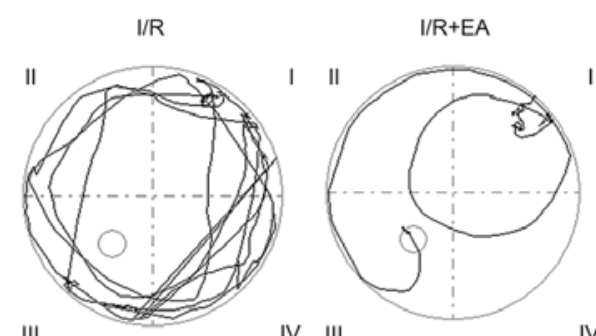

IV III

IV
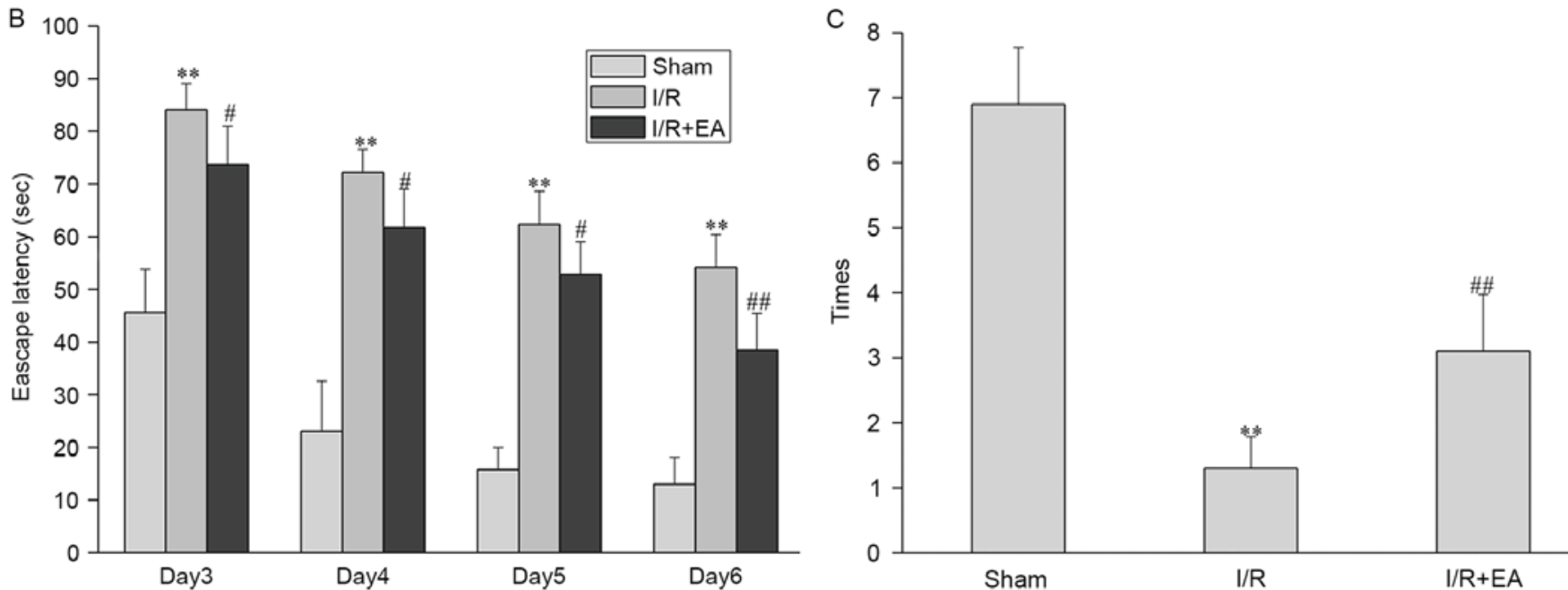

Figure 2. Effects of EA on cognitive impairment. (A) Representative tracing images from the Morris water maze test of Sham, I/R and I/R+EA groups (n=10). (B) Escape latency (duration of finding the platform within $90 \mathrm{sec}$ ). (C) Number of times the rats passed through the area in which the platform was located. ${ }^{* *} \mathrm{P}<0.01$ vs. the Sham group; ${ }^{*} \mathrm{P}<0.05,{ }^{\# \#} \mathrm{P}<0.01$ vs. the I/R group. I/R, ischemia-reperfusion; EA, electro-acupuncture. 


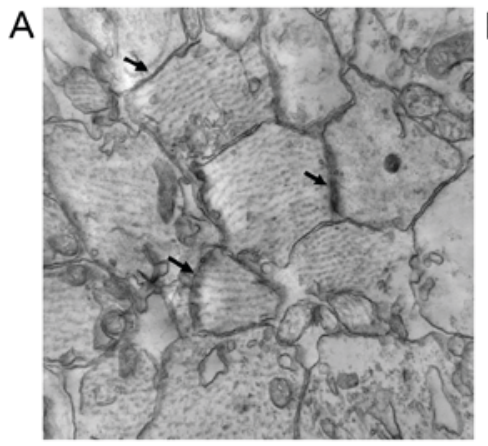

Sham

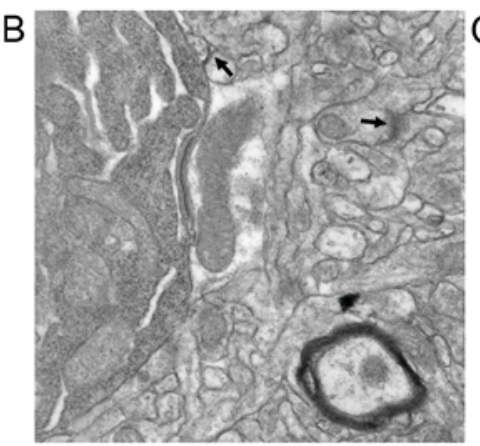

$\mathbf{I} / \mathbf{R}$

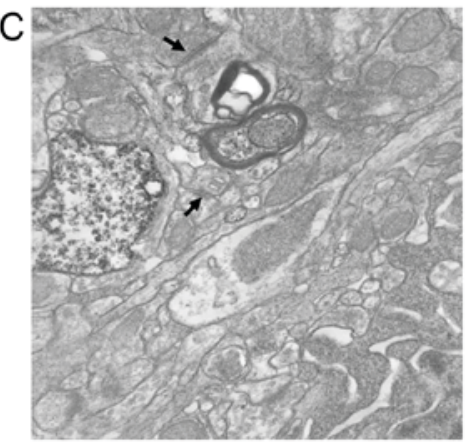

$\mathbf{I} / \mathbf{R}+\mathbf{E A}$

Figure 3. Effect of EA on synaptic structural plasticity. Electron microscopy images indicating the changes to synaptic ultrastructure (indicated by arrows) of the hippocampus in the (A) Sham, (B) I/R and (C) I/R+EA groups (magnification, $x 50,000 ; n=5$ ). I/R, ischemia-reperfusion; EA, electro-acupuncture.

A

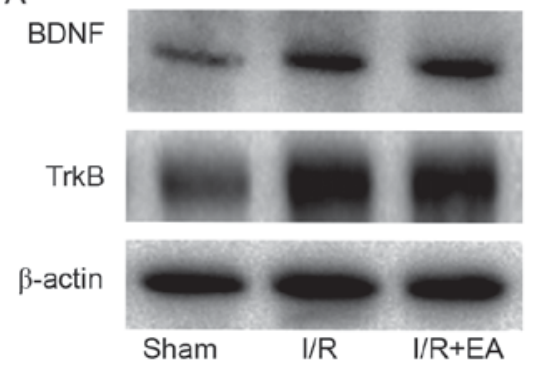

B

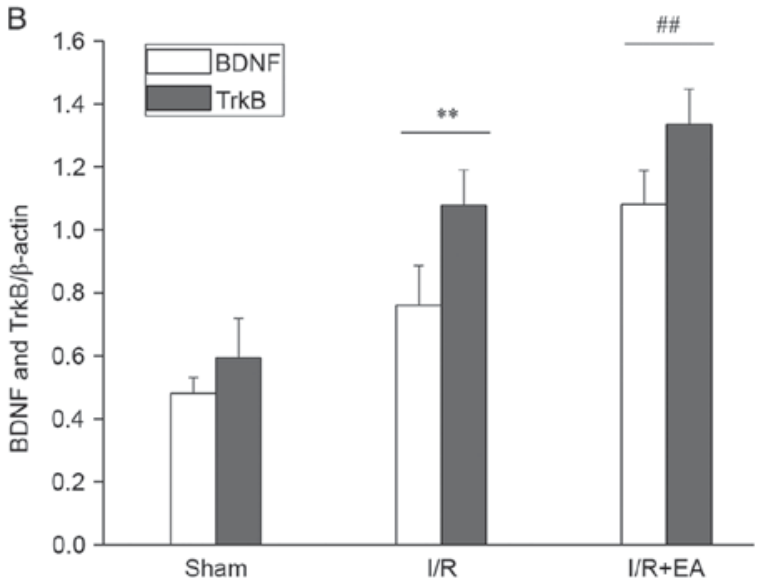

Figure 4. Effect of EA on BDNF and TrkB. (A) Western blot showing the levels of BDNF and TrkB in the hippocampus of Sham, I/R and I/R+EA groups $(\mathrm{n}=5)$. (B) Bar graph showing the quantified expression levels of BDNF and TrkB from each group. ${ }^{* *} \mathrm{P}<0.01$ vs. the Sham group; ${ }^{\# \#} \mathrm{P}<0.01$ vs. the I/R group. I/R, ischemia-reperfusion; EA, electro-acupuncture; BDNF, brain-derived neurotrophic factor; TrkB, tropomyosin receptor kinase B.

Such cognitive improvement is indicative of the hippocampal structural and functional synaptic plasticity in the ipsilateral hemisphere, including the expression of hippocampal BDNF, TrkB and PSD-95. The phenomena of long-term potentiation (LTP) and long-term depression (LTD) have been acknowledged as the activity level of cell biological basis of learning and memory. Therefore, plasticity is decreased in post-stroke cognitive impairment. EA may enhance the LTP and inhibit the LTD of this plasticity, delaying cognitive repair. BDNF would affect the neuronal growth, survival, differentiation

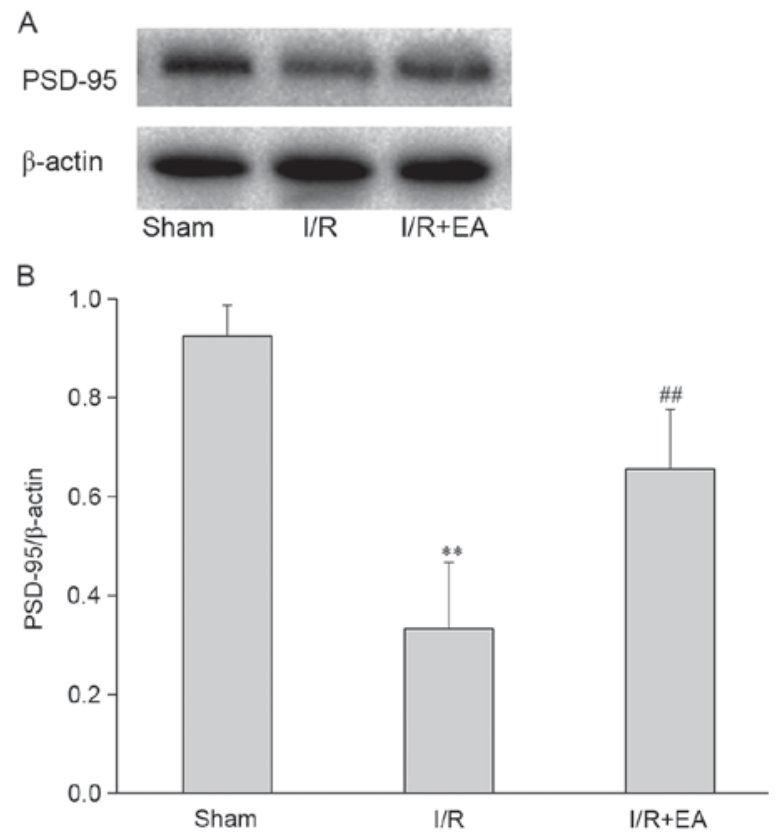

Figure 5. Effect of EA on PSD-95. (A) Western blot showing the levels of PSD-95 in the hippocampus of the Sham, I/R and I/R+EA groups $(n=5)$. (B) Bar graph showing the quantified expression levels of PSD-95 from each group. ${ }^{* *} \mathrm{P}<0.01$ vs. the Sham group; ${ }^{\# \#} \mathrm{P}<0.01$ vs. the I/R group. I/R, ischemia-reperfusion; EA, electro-acupuncture; PSD-95, post-synaptic density protein-95.

and development, and it serves an important role, repairing the neuronal damage (22). The immature prBDNF and mature mBDNF are the two primary forms of BDNF (23). BDNF, specifically mBDNF on TrkB with high affinity, works by phosphorylating TrkB. BDNF stimulates regeneration of synapses. It is therefore speculated that the expression of BDNF and the TrkB of the hippocampus promote phosphorylation, then raise the synapse in the hippocampus, indicated by the increased expression of BDNF and TrkB, and promote the regeneration of the neural spines protrusions by electroacupuncture activation, which serves a role in the treatment of ischemia reperfusion memory impairment.

The results of the present study showed that EA treatment at the Shenting (DU24) and Baihui (DU20) acupoints ameliorated neurological deficits and reduced cerebral infarct volumes. Consistent with the findings of previous studies by 
our group (7,8), a Morris water maze test demonstrated that EA improved the learning and memory ability of rats with cerebral I/R injury, confirming the therapeutic efficacy of EA on post-stroke cognitive impairment.

The hippocampus is crucially important for learning and memory, and is the focus of numerous studies on normal function and pathological models of disease $(24,25)$. The synapse is the functional unit of brain (26). Synaptic structural integrity has a vital role in memory storage (27). The present study found that in the I/R group, only small amounts of dendrites and axons were present, with the synapse structure disordered and damaged. By contrast, in the I/R+EA group, synaptic clefts were identifiable and certain synaptic structures were similar to those in the Sham group, which showed that EA promotes synaptic structural plasticity.

Besides structural aspects of plasticity induced by EA, synapse function may have also been enhanced as indirectly supported by the increase of BDNF and its receptor TrkB. For instance, numerous studies have confirmed that BDNF and TrkB are involved in long-term potentiation by using BDNF and TrkB knockout mice (28-30).

Regarding synaptic protein concentrations, EA treatment at the Shenting (DU24) and Baihui (DU20) acupoints was observed to enhance the expression of hippocampal BDNF, TrkB and PSD-95 in the ipsilateral hemisphere. PSD-95 is a neuronal PDZ protein that associates with receptors and cytoskeletal elements at synapses (31). In the present study, the levels of PSD-95 were possibly regulated by BDNF, since certain studies reported the principal role of BDNF in synaptic plasticity and learning and memory processes (32-34). Inhibition of BDNF signaling has been previously shown to decrease hippocampal PSD-95 expression and dendritic growth (35). Furthermore, BDNF and its TrkB receptor may also make a great contribution to facilitating glutamate receptor stabilization through PSD-95 for excitatory synapse formation (31).

In conclusion, the present study indicated a positive effect of EA on Shenting (DU24) and Baihui (DU20) acupoints, which contributed to the improvement of cognitive function after cerebral I/R injury. The possible underlying mechanism, which comprises the enhancement of synaptic plasticity by BDNF and its TrkB receptor during EA treatment, was also elucidated. These data suggested that EA is a promising approach for treating post-stroke cognitive impairment. However, the long-term effect of EA treatment on Shenting (DU24) and Baihui (DU20) acupoints is yet to be determined.

\section{Acknowledgements}

The present study was supported by the National Natural Science Foundation of China (grant no. 81574048), the Natural Science Foundation of Fujian Province (grant no. 2015J01335), the Fujian Rehabilitation Tech Co-innovation Center, the Fujian Key Laboratory of Rehabilitation Technology and the Fujian Provincial Rehabilitation Industrial Institution.

\section{References}

1. Roger VL, Go AS, Lloyd-Jones DM, Adams RJ, Berry JD, Brown TM, Carnethon MR, Dai S, de Simone G, Ford ES, et al: Heart disease and stroke statistics-2011 update: A report from the American Heart Association. Circulation 123: e18-e209, 2011.
2. Alvarez-Sabin J and Román GC: Citicoline in vascular cognitive impairment and vascular dementia after stroke. Stroke 42 (Suppl 1): S40-S43, 2011.

3. Haring HP: Cognitive impairment after stroke. Curr Opin Neurol 15: 79-84, 2002.

4. Mok VC, Wong A, Lam WW, Fan YH, Tang WK, Kwok T, Hui AC and Wong KS: Cognitive impairment and functional outcome after stroke associated with small vessel disease. J Neurol Neurosurg Psychiatry 75: 560-566, 2004.

5. Pasi M, Poggesi A, Salvadori E and Pantoni L: Post-stroke dementia and cognitive impairment. Front Neurol Neurosci 30: 65-69, 2012.

6. Zheng G, Liu F, Li S, Huang M, Tao J and Chen L: Tai Chi and the protection of cognitive ability: A systematic review of prospective studies in healthy adults. Am J Prev Med 49: 89-97, 2015.

7. Feng X, Yang S, Liu J, Huang J, Peng J, Lin J, Tao J and Chen L: Electroacupuncture ameliorates cognitive impairment through inhibition of NF- $\mathrm{KB}$-mediated neuronal cell apoptosis in cerebral ischemia-reperfusion injured rats. Mol Med Rep 7: 1516-1522, 2013.

8. Lin R, Lin Y, Tao J, Chen B, Yu K, Chen J, Li X and Chen LD: Electroacupuncture ameliorates learning and memory in rats with cerebral ischemia-reperfusion injury by inhibiting oxidative stress and promoting p-CREB expression in the hippocampus. Mol Med Rep 12: 6807-6814, 2015.

9. Gutiérrez-Vargas JA, Múnera A and Cardona-Gómez GP: CDK5 knockdown prevents hippocampal degeneration and cognitive dysfunction produced by cerebral ischemia. J Cereb Blood Flow Metab 35: 1937-1949, 2015.

10. Vaynman S, Ying Z and Gómez-Pinilla F: Exercise induces BDNF and synapsin I to specific hippocampal subfields. J Neurosci Res 76: 356-362, 2004.

11. Adasme T, Haeger P, Paula-Lima AC, Espinoza I, Casas-Alarcón MM, Carrasco MA and Hidalgo C: Involvement of ryanodine receptors in neurotrophin-induced hippocampal synaptic plasticity and spatial memory formation. Proc Natl Acad Sci USA 108: 3029-3034, 2011.

12. Prosser-Loose EJ, Verge VM, Cayabyab FS and Paterson PG: Protein-energy malnutrition alters hippocampal plasticity-associated protein expression following global ischemia in the gerbil. Curr Neurovasc Res 7: 341-360, 2010.

13. Huang L, Applegate PM, Gatling JW, Mangus DB, Zhang J and Applegate RL II: A systematic review of neuroprotective strategies after cardiac arrest: From bench to bedside (part II-comprehensive protection). Med Gas Res 4: 10, 2014.

14. Berretta A, Tzeng YC and Clarkson AN: Post-stroke recovery: The role of activity-dependent release of brain-derived neurotrophic factor. Expert Rev Neurother 14: 1335-1344, 2014.

15. Ho VM, Lee JA and Martin KC: The cell biology of synaptic plasticity. Science 334: 623-628, 2011.

16. Kiprianova I, Sandkühler J, Schwab S, Hoyer S and Spranger M: Brain-derived neurotrophic factor improves long-term potentiation and cognitive functions after transient forebrain ischemia in the rat. Exp Neurol 159: 511-519, 1999.

17. Shih PC, Yang YR and Wang RY: Effects of exercise intensity on spatial memory performance and hippocampal synaptic plasticity in transient brain ischemic rats. PLoS One 8: e78163, 2013.

18. Yi W, Xu NG and Wang GB: Experimental study on effects of electro-acupuncture in improving synaptic plasticity in focal cerebral ischemia rats. Zhongguo Zhong Xi Yi Jie He Za Zhi 26: 710-714, 2006 (In Chinese).

19. Longa EZ, Weinstein PR, Carlson S and Cummins R: Reversible middle cerebral artery occlusion without craniectomy in rats. Stroke 20: 84-91, 1989.

20. Bederson JB, Pitts LH, Tsuji M, Nishimura MC, Davis RL and Bartkowski H: Rat middle cerebral artery occlusion: Evaluation of the model and development of a neurologic examination. Stroke 17: 472-476, 1986.

21. Pouzet B, Zhang WN, Feldon J and Rawlins JN: Hippocampal lesioned rats are able to learn a spatial position using non-spatial strategies. Behav Brain Res 133: 279-291, 2002.

22. Wan D, Zhu HF, Luo Y and Xie P: Catalpol up-regulates expression of BDNF and TrkB proteins within the peri-infarct cortex in rats with focal cerebral ischemia . Chin Pharmacol Bull 27: 787-792, 2013.

23. Hashimoto K, Shimizu E and Iyo M: Critical role of brain-derived neurotrophic factor in mood disorders. Brain Res Brain Res Rev 45: 104-114, 2004.

24. Deng W, Aimone JB and Gage FH: New neurons and new memories: How does adult hippocampal neurogenesis affect learning and memory? Nat Rev Neurosci 11: 339-350, 2010. 
25. Manns JR and Eichenbaum H: A cognitive map for object memory in the hippocampus. Learn Mem 16: 616-624, 2009.

26. Mayford M, Siegelbaum SA and Kandel ER: Synapses and memory storage. Cold Spring Harb Perspect Biol 4: pii: a005751, 2012.

27. Bailey $\mathrm{CH}$ and Kandel ER: Structural changes accompanying memory storage. Annu Rev Physiol 55: 397-426, 1993.

28. Korte M, Carroll P, Wolf E, Brem G, Thoenen H and Bonhoeffer T: Hippocampal long-term potentiation is impaired in mice lacking brain-derived neurotrophic factor. Proc Natl Acad Sci USA 92: 8856-8860, 1995.

29. Patterson SL, Abel T, Deuel TA, Martin KC, Rose JC and Kandel ER: Recombinant BDNF rescues deficits in basal synaptic transmission and hippocampal LTP in BDNF knockout mice. Neuron 16: 1137-1145, 1996

30. Minichiello L, Korte M, Wolfer D, Kühn R, Unsicker K, Cestari V, Rossi-Arnaud C, Lipp HP, Bonhoeffer T and Klein R: Essential role for TrkB receptors in hippocampus-mediated learning. Neuron 24: 401-414, 1999.
31. El-Husseini AE, Schnell E, Chetkovich DM, Nicoll RA and Bredt DS: PSD-95 involvement in maturation of excitatory synapses. Science 290: 1364-1368, 2000.

32. Chuang CM, Hsieh CL, Lin HY and Lin JG: Panax Notoginseng Burk attenuates impairment of learning and memory functions and increases ED1, BDNF and beta-secretase immunoreactive cells in chronic stage ischemia-reperfusion injured rats. Am J Chin Med 36: 685-693, 2008.

33. Gómez-Palacio Schjetnan A and Escobar-Rodriguez ML: Memory coding and retention: Brain-derived neurotrophic factor (BDNF) in synaptic plasticity. Rev Neurol 45: 409-417, 2007.

34. Waterhouse EG and $\mathrm{Xu} \mathrm{B}$ : New insights into the role of brain-derived neurotrophic factor in synaptic plasticity. Mol Cell Neurosci 42: 81-89, 2009.

35. Charych EI, Akum BF, Goldberg JS, Jörnsten RJ, Rongo C, Zheng JQ and Firestein BL: Activity-independent regulation of dendrite patterning by postsynaptic density protein PSD-95. J Neurosci 26: 10164-10176, 2006. 